LETTERS

\title{
Experimental evolution of bet hedging
}

\author{
Hubertus J. E. Beaumont ${ }^{1,2} \dagger$, Jenna Gallie ${ }^{1}$, Christian Kost $^{1} \dagger$, Gayle C. Ferguson ${ }^{1} \&$ Paul B. Rainey ${ }^{1}$
}

\begin{abstract}
Bet hedging - stochastic switching between phenotypic states ${ }^{1-3}$ is a canonical example of an evolutionary adaptation that facilitates persistence in the face of fluctuating environmental conditions. Although bet hedging is found in organisms ranging from bacteria to humans ${ }^{4-10}$, direct evidence for an adaptive origin of this behaviour is lacking ${ }^{11}$. Here we report the de novo evolution of bet hedging in experimental bacterial populations. Bacteria were subjected to an environment that continually favoured new phenotypic states. Initially, our regime drove the successive evolution of novel phenotypes by mutation and selection; however, in two (of 12) replicates this trend was broken by the evolution of bet-hedging genotypes that persisted because of rapid stochastic phenotype switching. Genome re-sequencing of one of these switching types revealed nine mutations that distinguished it from the ancestor. The final mutation was both necessary and sufficient for rapid phenotype switching; nonetheless, the evolution of bet hedging was contingent upon earlier mutations that altered the relative fitness effect of the final mutation. These findings capture the adaptive evolution of bet hedging in the simplest of organisms, and suggest that riskspreading strategies may have been among the earliest evolutionary solutions to life in fluctuating environments.
\end{abstract}

Life exists in ever-changing environments, but surviving under fluctuating conditions poses challenges. One solution is the evolution of mechanisms that allow modulation of phenotype in response to specific environmental cues. An alternative solution is stochastic phenotype switching, a strategy based on bet hedging, rather than direct environmental sensing ${ }^{4-10}$.

The general prediction from theory is that fluctuating selection generated by unpredictable environments can favour the evolution of bet hedging ${ }^{1-4,12-14}$. Under such conditions, a strategy that generates random variation in fitness-related traits among individuals within a population can enhance long-term fitness by increasing the likelihood that a subset of individuals expresses a phenotype that will be adaptive in a future environment ${ }^{3,15,16}$. However, the outcome of adaptive evolution under fluctuating selection is also shaped by factors such as the frequency of environmental change ${ }^{12}$, the capacity of a given population to respond to fluctuations by mutation and selection (that is, evolvability), the presence of suitable environmental cues ${ }^{13}$ and the cost-benefit balance of different strategies ${ }^{12}$.

Here we report the de novo evolution of bet hedging in experimental bacterial populations. Our populations experienced repeated bouts of selection in two contrasting environments; they also experienced fluctuating selection wrought by imposition of an exclusion rule and population bottleneck. Applied at the point of transfer between environments, the exclusion rule assigned a fitness of zero to the type that was common in the current environment; imposition of the bottleneck meant that only a single phenotypically distinct type was selected from among the survivors to found the next bout of selection. The exclusion rule imposed strong selection for phenotypic innovation, whereas the bottleneck negated the cost of bet hedging - that is, the generation of types maladapted to the prevailing conditions - by eliminating competition with conspecifics. A natural analogue of this mode of fluctuating selection is imposed by the host immune system on invading microorganisms. Indeed, many pathogens have evolved bet-hedging strategies based on stochastic antigen switching ${ }^{8}$.

When the ancestral genotype of Pseudomonas fluorescens SBW25 is grown in static broth microcosms, it rapidly diversifies into a range of niche specialist genotypes by mutation and selection, which each form distinct colonies on agar plates ${ }^{17}$. In contrast, diversification is constrained in shaken microcosms, which favour genotypes with an ancestral colony type $^{18}$. We exploited the pleiotropic correlation between niche specialization and colony morphology to realize our selection regime. The expected evolutionary response was repeated evolution, fixation and extinction of genotypes with novel colony morphologies.

Twelve replicate selection lines were founded with the ancestral genotype and subjected to 16 rounds of alternating selection in static and shaken microcosms. During each round, populations were propagated by serial dilution until the emergence of cells that formed colonies with a heritable morphology different from that of their immediate ancestor. Selection for different colonies was open-ended rather than for an a priori defined morphology. Once detected, cells derived from a single individual of this new type were transferred to the opposing environment for the next round of selection (Fig. 1a and Methods). Thus, we imposed selection for a high growth rate in static and shaken microcosms, and simultaneously fluctuating selection for colony innovation. Each lineage was associated with a cognate control line that was under stabilizing selection for the ancestral colony morphology, but otherwise treated identically (see Methods).

This selection regime indeed drove the repeated evolution of new colony morphologies. However, here we concentrate on the evolution of genotypes capable of rapid, stochastic colony-morphology switching (hereafter referred to as colony switching), which emerged in two of 12 replicates $\left(1 \mathrm{~B}^{4}\right.$ and $6 \mathrm{~B}^{4}$, Supplementary Note 1$)$. Each of these switching genotypes formed distinct translucent and opaque colonies (Fig. 1b). Colony switching persisted in both lines for seven additional rounds of selection, after which the experiment was ended. None of the control lines gave rise to colony switching.

To obtain insight into the mechanisms by which switching types persisted, we re-imposed our selection regime on the genotype $1 \mathrm{~B}^{4}$ (Fig. 1b), but this time ignored the colony variants it generated through colony switching. Without exception, $1 \mathrm{~B}^{4}$ was driven to extinction by the evolution of new genotypes with novel colony morphologies, some of which did not switch (Supplementary Note 2). This shows that persistence was attributable to bet hedging - that is, the capacity to generate at high-frequency colonies that were phenotypically distinct from those selected in the previous round-rather than to an intrinsic growth rate advantage over non-switching types. This finding also draws attention to the importance of the bottleneck: in its absence,

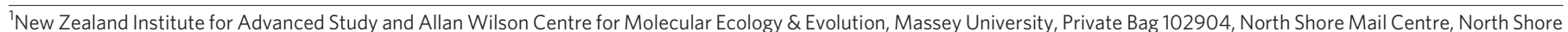

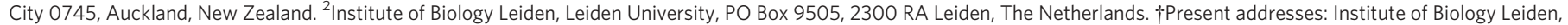

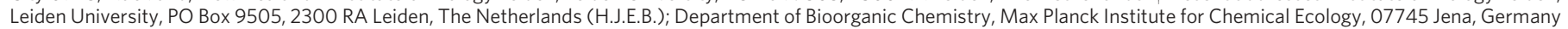
(C.K.). 
a
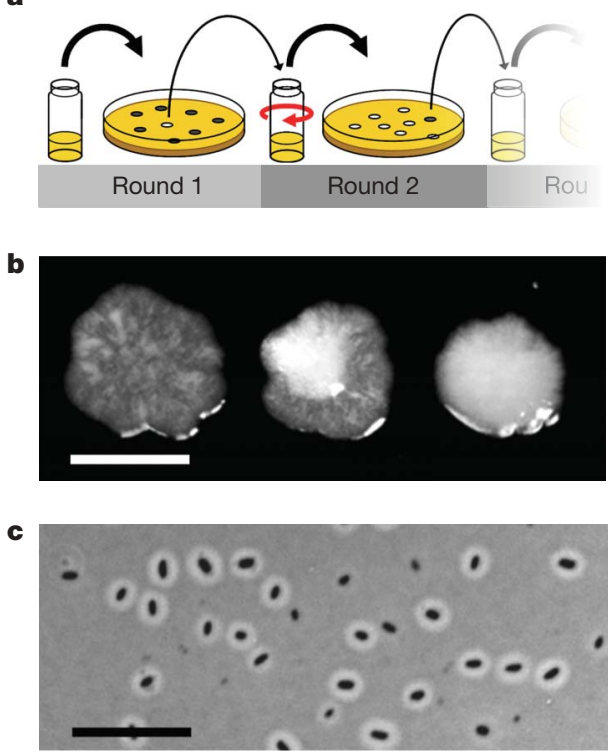

Figure 1 | Colony morphology evolution. a, Populations were propagated in static or shaken (red arrow) microcosms, and periodically screened for cells that form novel colony types, a prerequisite for passage to the opposing environment (the exclusion rule). Propagation between environments occurred by transfer of cells taken from a single, numerically dominant, novel colony (the bottleneck). b, From left: translucent, sectored and opaque colonies of a bet-hedging genotype $\left(1 \mathrm{~B}^{4}\right)$. Sectored colonies, which resulted from stochastic capsule-expression switching during formation of the colony, were counted as opaque. Scale bar, approximately $2 \mathrm{~mm}$. c, Capsulated and non-capsulated cells of $1 \mathrm{~B}^{4}$ (phase-contrast light microscopy with negative capsule staining). The proportions of capsulated cells in colonies produced by the original ancestor $\left(1 \mathrm{~A}^{0}\right)$ and immediate ancestor $\left(1 \mathrm{~A}^{4}\right)$ of $1 \mathrm{~B}^{4}$ were three orders of magnitude lower $(n=5,500$ cells examined per colony, binomial 95\% confidence intervals $0.0010-0.0037$ and $0.0028-0.0057$, respectively). Scale bar, approximately $10 \mu \mathrm{m}$.

$1 \mathrm{~B}^{4}$ would have been eliminated by non-switching genotypes with faster growth rates.

The serendipitous discovery that centrifugation of $1 \mathrm{~B}^{4}$ cells resulted in two discrete fractions prompted microscopic examination (Supplementary Method 2), which revealed that $1 \mathrm{~B}^{4}$ produced both capsulated $\left(\mathrm{Cap}^{+}\right)$and non-capsulated $\left(\mathrm{Cap}^{-}\right)$cells (Fig. 1c). Opaque colonies contained a higher proportion of $\mathrm{Cap}^{+}$cells than translucent colonies (Fig. 2a). When cell suspensions were plated from either colony type, both gave rise to a mixture of opaque and translucent colonies; however, cell suspensions made from opaque
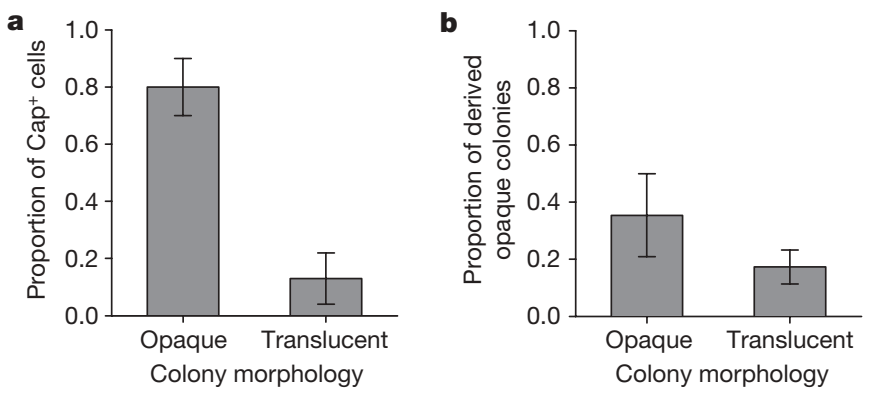

Figure 2 | Link between colony and cell morphology. a, Mean proportion of capsulated cells in opaque and translucent colonies of bet-hedging genotype $1 \mathrm{~B}^{4}(n=6,100$ cells examined per colony). Opaque colonies contained proportionally more capsulated cells than translucent colonies (analysis of deviance, $F(1,10)=61.795, P<0.0001)$. Error bars, one standard deviation. b, Mean proportion of opaque colonies formed by cell suspensions prepared from the opaque and translucent colonies in a $(n=6$, between 200 and 700 colonies examined). Cells derived from opaque colonies formed proportionally more opaque colonies (analysis of deviance, $F(1,10)=11.836, P=0.0063)$. Error bars, one standard deviation. colonies produced a higher proportion of opaque colonies than those derived from translucent colonies (Fig. 2b). Together, this suggests that the founding cell of a colony may determine the morphology of the latter by biasing the ratio of $\mathrm{Cap}^{+}$and $\mathrm{Cap}^{-}$cells. This causal link was corroborated by transposon mutagenesis ${ }^{19}$, which also indicated that the capsules consist of colanic acid ${ }^{20}$, a previously described capsule polysaccharide (see Supplementary Method 1 and Supplementary Table 1). Colony switching in the second bet-hedging genotype also involved $\mathrm{Cap}^{+}$and $\mathrm{Cap}^{-}$cells.

A likely mechanism of colony switching is reversible on-off switching of capsule production. Alternatively, colony switching may involve distinct non-switching $\mathrm{Cap}^{+}$and $\mathrm{Cap}^{-}$genotypes that form mixed colonies upon plating owing to chance co-localization, or mixed cell-type aggregates. We distinguished between these possibilities by determining whether populations that were passed through two sequential single-cell bottlenecks produced both $\mathrm{Cap}^{+}$and Cap cells after each bottleneck (see Supplementary Method 2 and Supplementary Fig. 1). All replicate populations generated both cell types after each bottleneck. The probability of observing this pattern under the null hypotheses of no reversible switching is exceedingly low (one-tailed Fisher's exact test, $P=0.0004$; see Supplementary Note 3 ), indicating that $1 \mathrm{~B}^{4}$ switches reversibly between $\mathrm{Cap}^{+}$and $\mathrm{Cap}^{-}$.

Although bet hedging facilitated the long-term persistence of $1 \mathrm{~B}^{4}$, the evolutionary emergence of this genotype required it to reach a detectable frequency within a selection round. To examine if $1 \mathrm{~B}^{4}$ owed its emergence to a higher fitness than its immediate ancestor $\left(1 \mathrm{~A}^{4}\right)$, we competed these two genotypes in static microcosms, the environment in which $1 \mathrm{~B}^{4}$ emerged. This indicated that $1 \mathrm{~B}^{4}$ was indeed more fit than $1 \mathrm{~A}^{4}$ (one-sample $t$-test, $n=8, P=0.0002$; Fig. 4 ).

Repeated single-cell bottlenecks can drive the fixation of random deleterious mutations, causing a decline in fitness. To test if this had occurred during our experiment, we measured the fitness of all genotypes in the $1 \mathrm{~B}^{4}$ lineage relative to the original ancestor in both static and shaken microcosms (Fig. 3a). This revealed no evidence for a decrease in fitness. Interestingly, the results indicate non-transitive fitness interactions between some consecutive genotypes (for example, $1 \mathrm{~A}^{4}$ is more fit than $1 \mathrm{~B}^{4}$ relative to $1 \mathrm{~A}^{0}$, but less fit than $1 \mathrm{~B}^{4}$ during direct competition).

Using whole-genome re-sequencing ${ }^{21,22}$, the entire 6.7-megabasepair genome of $1 \mathrm{~B}^{4}$ (ref. 23) was analysed to unravel its mutational history. Nine mutations separating $1 \mathrm{~B}^{4}$ from the original ancestor were identified, confirmed by Sanger sequencing, and ordered by inspection of the affected loci in the preceding genotypes (Fig. 3b). With the exception of the final mutation, all mutations involved nonsynonymous changes at loci previously demonstrated to be mutational targets in the evolution of wrinkly spreader types ${ }^{18,24-26}$ (Fig. 3b). The final mutation was a single non-synonymous nucleotide change in carB (Arg674Cys, Fig. 3b), which encodes the large subunit of carbamoylphosphate synthetase (CarAB, EC 6.3.5.5), a central enzyme of the pyrimidine and arginine biosynthetic pathways ${ }^{27}$.

To examine the causal connection between the carB mutation and colony switching, we introduced this mutation in the immediate ancestor $\left(1 \mathrm{~A}^{4}\right)$ by allelic replacement. The engineered genotype displayed colony switching (Fig. $3 \mathrm{~g}$ ). Conversely, reversion of the carB mutation in $1 \mathrm{~B}^{4}$ to wild type abolished colony switching (Fig. $3 \mathrm{~h}$ ). This demonstrates that the carB mutation is sufficient and necessary to cause stochastic colony morphology switching in $1 \mathrm{~A}^{4}$.

Although the exact mechanism of colony switching remains to be elucidated, two lines of evidence suggest that switching might be controlled epigenetically, rather than by a mutable locus ${ }^{8}$. First, $\mathrm{Cap}^{+}$and Cap ${ }^{-}$cells were identical at the carB locus. Second, neither transposon mutagenesis nor genome re-sequencing showed evidence of the involvement of mutable loci (Supplementary Method 1).

If the $\operatorname{carB}$ mutation is the sole cause of the evolution of colony switching from $1 \mathrm{~A}^{4}$, it must confer not only colony switching but also the requisite high fitness in static microcosms in this genetic background. To assess this, we introduced the mutant carB allele in $1 \mathrm{~A}^{4}$ 


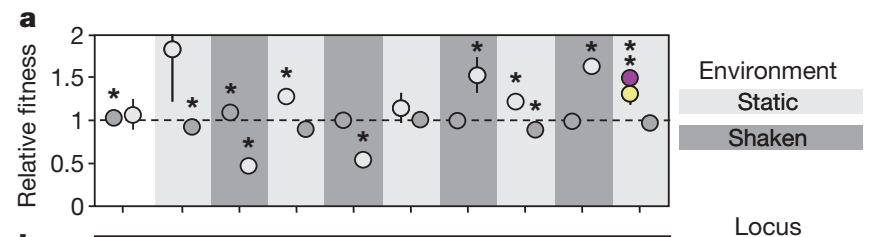

b

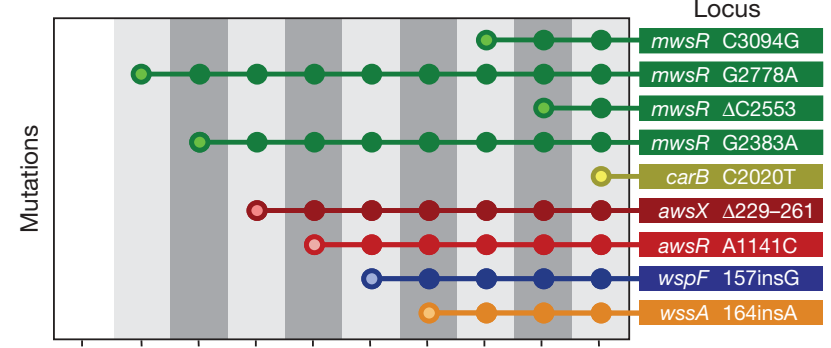

$1 A^{0}>1 B^{0}>1 A^{1}>1 B^{1}>1 A^{2}>1 B^{2}>1 A^{3}>1 B^{3}>1 A^{4}>1 B^{4}$

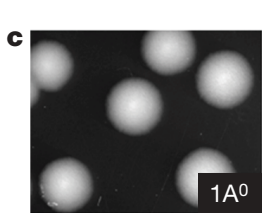

$$
\text { Genotype }
$$
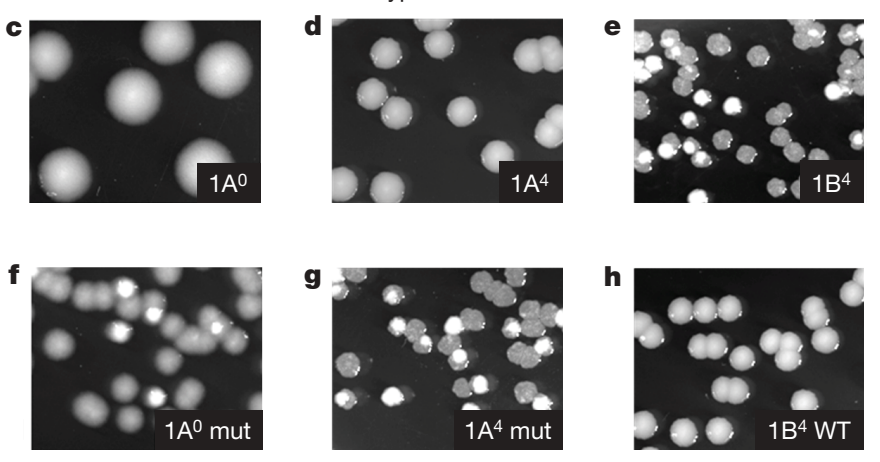

Figure 3 | Fitness, mutational history and effects of the carB mutation in a bet-hedging lineage. $a$, Fitness of each genotype in a bet-hedging lineage $\left(1 \mathrm{~B}^{4}\right)$ measured relative to the original ancestor $\left(1 \mathrm{~A}^{0}\right)$ in shaken (dark circles) and static (light circles) microcosms. Light bars, genotypes that evolved in static microcosms $\left(1 \mathrm{~B}^{n}\right)$; dark bars, genotypes that evolved in shaken microcosms $\left(1 \mathrm{~A}^{n}\right)$. Fitness of $1 \mathrm{~B}^{4}$ in static microcosms founded with predominantly capsulated (yellow circles, Cap ${ }^{+}$proportion $=0.688,95 \%$ confidence interval $0.417-0.872$ ) or non-capsulated cells (purple circles, $\mathrm{Cap}^{+}$proportion $=0.013,95 \%$ confidence interval 0.004-0.031) did not differ significantly. Asterisks, significant deviation from 1 (dashed line; $P \leq 0.05$, one-sample $t$-tests, $n=3$ ). Error bars, one standard deviation. b, Mutations identified by genome re-sequencing of $1 \mathrm{~B}^{4}$ and ordered through analysis of the preceding genotypes. Each round of selection caused a genetic change readily explained by existing knowledge of the genetic causes of the WS phenotype ${ }^{18,24-26} \cdot \mathbf{c}-\mathbf{h}$, Colonies of (c) original ancestral genotype, (d) immediate ancestor of bet-hedging genotype, (e) bet-hedging genotype, (f) original ancestral genotype with mutated $\operatorname{carB},(\mathbf{g})$ immediate ancestor of bet-hedging genotype with mutated $c a r B$ and (h) bet-hedging genotype with wild-type carB. Colonies were photographed at the same magnification.

and competed the resulting genotype against $1 \mathrm{~A}^{4}$. This showed that the carB mutation increases the fitness of $1 \mathrm{~A}^{4}$ (one-sample $t$-test, $n=9, P=0.0013$; Fig. 4). Moreover, $1 \mathrm{~B}^{4}$ with a wild-type carB allele had a lower fitness relative to $1 \mathrm{~B}^{4}$, confirming that the carB mutation was essential for the increased fitness of this genotype (one-sample $t$-test, $n=9, P=0.0004$; Fig. 4). Together, these results indicate that the fitness effect of the carB mutation is sufficient to explain the emergence of $1 \mathrm{~B}^{4}$ to a detectable frequency.

Colony switching was caused by a single point mutation but nonetheless took nine rounds of selection to evolve. This led us to question the importance of the previously fixed mutations ${ }^{28}$. To study this, one round of the evolutionary experiment was repeated from both $1 \mathrm{~A}^{4}$ and the original ancestor $\left(1 \mathrm{~A}^{0}\right)$. Colony switching evolved from $1 \mathrm{~A}^{4}$ ( 3 from 36 replicates), but never from the ancestral genotype ( 0 from 138 replicates), indicating that these genotypes differed in their capacity to give rise to colony switching (two-tailed Fisher's exact test, $P=0.0083$ ).

The reliance on previously fixed mutations might stem from epistatic interactions essential for the carB mutation to cause colony switching or to confer the requisite fitness benefit in static microcosms.

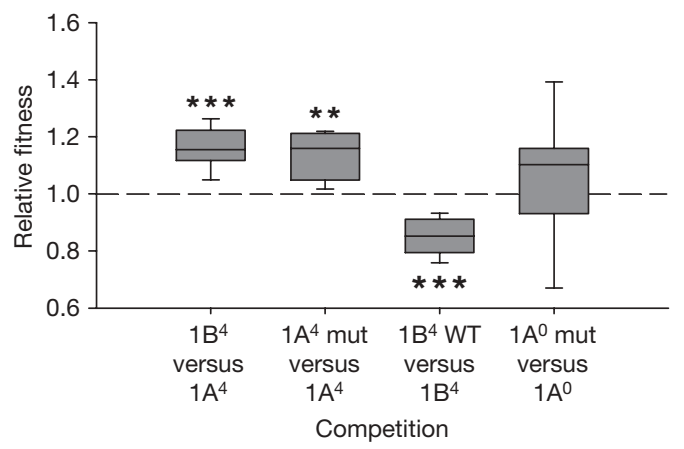

Figure 4 | Relative fitness of evolved and engineered strains. Box plot of the fitness of a bet-hedging genotype relative to its immediate ancestor $\left(1 \mathrm{~B}^{4}\right.$ versus $1 \mathrm{~A}^{4}$ ), and the fitness effects of mutated carB in the immediate ancestor of the bet-hedging genotype $\left(1 \mathrm{~A}^{4}\right.$ mut versus $\left.1 \mathrm{~A}^{4}\right)$, wild-type carB in $1 \mathrm{~B}^{4}\left(1 \mathrm{~B}^{4} \mathrm{WT}\right.$ versus $\left.1 \mathrm{~B}^{4}\right)$ and mutated carB in the original ancestor $\left(1 \mathrm{~A}^{0} \mathrm{mut}\right.$ versus $\left.1 \mathrm{~A}^{0}\right)$. All fitness assays were performed in static microcosms. Values greater than 1 (dashed line) indicate a higher relative fitness of the first competitor. Key: median (horizontal lines in boxes), interquartile range (boxes), 90th and 10th percentiles (vertical bars), significant deviation from 1 $\left(* * * P \leq 0.001,{ }^{* *} P \leq 0.01\right.$; see text for statistics).

We distinguished between these hypotheses by introducing the car $B$ mutation in $1 \mathrm{~A}^{0}$. In this background it did cause colony switching (Fig. 3f) but appeared not to confer a significant fitness increase (Fig. 4). The latter was confirmed by a direct statistical comparison with the effect of the carB mutation in $1 \mathrm{~A}^{4}$ (analysis of covariance, the larger variance of the $1 \mathrm{~A}^{0}$ data is explained by a covariate), which identified a significant epistatic interaction (analysis of deviance, $F(1,16)=8.536, P=0.01)$, and indicated that the $c a r B$ mutation is beneficial in $1 \mathrm{~A}^{4}$ but deleterious in $1 \mathrm{~A}^{0}$ (Supplementary Note 4 ). We conclude that the evolutionary history of $1 \mathrm{~A}^{4}$ 'set the stage' for the evolution of stochastic colony morphology switching by altering the relative fitness effect of the carB mutation.

Owing to the historical nature of the evolutionary process, the origins of most adaptive phenotypes are obscure ${ }^{11}$. Here we have provided a mechanistic account of the adaptive evolution of a widespread trait ${ }^{4-10}$. Bet hedging arose as an adaptation to fluctuating selection imposed by an exclusion rule and bottleneck, two population processes that are likely to play a key role in the evolution of stochastic phenotype switching in nature. Insight into the underlying molecular details reveals how evolution tinkered with central metabolism to generate a strategy that could reasonably-one might think-have taken tens of thousands of generations to evolve. The rapid and repeatable evolution of bet hedging during our experiment suggests it may have been among the earliest evolutionary solutions to life in variable environments, perhaps even preceding the evolution of environmentally responsive mechanisms of gene regulation.

\section{METHODS SUMMARY}

P. fluorescens SBW25 (ref. 23) was grown in glass microcosms containing liquid medium. During each selection round, populations were propagated by transfer of a sample to a fresh microcosm. Parallel with each transfer, populations were checked for the presence of cells that formed new colony types. Identification of a colony different from that with which the selection round had been started marked the end of a selection round. Cells from these colonies were stored, and used to found the next selection round in the opposing environment. This procedure was repeated 15 times. Control lines were continually selected in shaken microcosms and always propagated by transfer of cells from colonies of the ancestral type. Relative fitness was measured in competition assays, using colony morphology or a neutral marker ${ }^{29}$ to distinguish genotypes, and expressed as the ratio of Malthusian parameters ${ }^{30}$.

Full Methods and any associated references are available in the online version of the paper at www.nature.com/nature.

\section{Received 7 July; accepted 15 September 2009.}

1. Cohen, D. Optimizing reproduction in a randomly varying environment. J. Theor. Biol. 12, 119-129 (1966). 
2. Seger, J. \& Brockmann, H. J. in Oxford Surveys in Evolutionary Biology, Vol. 4 (eds Harvey, P. \& Partridge, L.) 182-211 (Oxford Univ. Press, 1987).

3. Slatkin, M. Hedging one's evolutionary bets. Nature 250, 704-705 (1974).

4. Ackermann, M. et al. Self-destructive cooperation mediated by phenotypic noise. Nature 454, 987-990 (2008)

5. Danforth, B. N. Emergence dynamics and bet hedging in a desert bee, Perdita portalis. Proc. Natl Acad. Sci. USA 266, 1985-1994 (1999).

6. Friedenberg, N. A. Experimental evolution of dispersal in spatiotemporally variable microcosms. Ecol. Lett. 6, 953-959 (2003).

7. Hairston, N. G. \& Munns, W. R. The timing of copepod diapause as an evolutionary stable strategy. Am. Nat. 123, 733-751 (1984).

8. Moxon, E. R., Rainey, P. B., Nowak, M. A. \& Lenski, R. E. Adaptive evolution of highly mutable loci in pathogenic bacteria. Curr. Biol. 4, 24-33 (1994).

9. Tonegawa, S. Somatic generation of antibody diversity. Nature 302, 575-581 (1983).

10. Venable, D. L. Bet hedging in a guild of desert annuals. Ecology 88, 1086-1090 (2007).

11. Gould, S. J. \& Lewontin, R. C. The spandrels of San Marco and the Panglossian paradigm: a critique of the adaptationist programme. Proc. R. Soc. Lond. B 205, 581-598 (1979).

12. Kussell, E. \& Leibler, S. Phenotypic diversity, population growth, and information in fluctuating environments. Science 309, 2075-2078 (2005).

13. Wolf, D. M., Vazirani, V. V. \& Arkin, A. P. Diversity in times of adversity: probabilistic strategies in microbial survival games. J. Theor. Biol. 234, 227-253 (2005).

14. Meyers, L. A. \& Bull, J. J. Fighting change with change: adaptive variation in an uncertain world. Trends Ecol. Evol. 17, 551-557 (2002).

15. Acar, M., Mettetal, J. T. \& van Oudenaarden, A. Stochastic switching as a survival strategy in fluctuating environments. Nature Genet. 40, 471-475 (2008).

16. Balaban, N. Q., Merrin, J., Chait, R., Kowalik, L. \& Leibler, S. Bacterial persistence as a phenotypic switch. Science 305, 1622-1625 (2004).

17. Rainey, P. B. \& Travisano, M. Adaptive radiation in a heterogeneous environment. Nature 394, 69-72 (1998)

18. Spiers, A. J., Kahn, S. G., Bohannon, J., Travisano, M. \& Rainey, P. B. Adaptive divergence in experimental populations of Pseudomonas fluorescens. I. Genetic and phenotypic bases of wrinkly spreader fitness. Genetics 161, 33-46 (2002).

19. Giddens, S. R. et al. Mutational activation of niche-specific genes provides insight into regulatory networks and bacterial function in a complex environment. Proc. Natl Acad. Sci. USA 104, 18247-18252 (2007)

20. Stevenson, G., Andrianopoulos, K., Hobbs, M. \& Reeves, P. R. Organization of the Escherichia coli K-12 gene cluster responsible for production of the extracellular polysaccharide colanic acid. J. Bacteriol. 178, 4885-4893 (1996).

21. Albert, T. J. et al. Mutation discovery in bacterial genomes: metronidazole resistance in Helicobacter pylori. Nature Methods 2, 951-953 (2005).

22. Bentley, D. R. et al. Accurate whole human genome sequencing using reversible terminator chemistry. Nature 456, 53-59 (2008).

23. Silby, M. W. et al. Genomic and genetic analyses of diversity and plant interactions of Pseudomonas fluorescens. Genome Biol. 10, R51 (2009).
24. Bantinaki, E. et al. Adaptive divergence in experimental populations of Pseudomonas fluorescens. III. Mutational origins of wrinkly spreader diversity. Genetics 176, 441-453 (2007).

25. Goymer, P. et al. Adaptive divergence in experimental populations of Pseudomonas fluorescens. II. Role of the GGDEF regulator WspR in evolution and development of the wrinkly spreader phenotype. Genetics 173, 515-526 (2006).

26. McDonald, M. J., Gehrig, S. M., Meintjes, P. L., Zhang, X. X. \& Rainey, P. B. Adaptive divergence in experimental populations of Pseudomonas fluorescens. IV. Genetic constraints guide evolutionary trajectories in a parallel adaptive radiation. Genetics (in the press).

27. Mergeay, M., Gigot, D., Beckman, J., Glansdorff, N. \& Pierard, A. Physiology and genetics of carbamoylphosphate synthesis in Escherichia coli K-12. Mol. Gen. Genet. 133, 299-316 (1974)

28. Blount, Z. D., Borland, C. Z. \& Lenski, R. E. Historical contingency and the evolution of a key innovation in an experimental population of Escherichia coli. Proc. Natl Acad. Sci. USA 105, 7899-7906 (2008).

29. Zhang, X. X. \& Rainey, P. B. Construction and validation of a neutrally-marked strain of Pseudomonas fluorescens SBW25. J. Microbiol. Methods 71, 78-81 (2007).

30. Lenski, R. E., Rose, M. R., Simpson, S. C. \& Tadler, S. C. Long-term experimental evolution in Escherichia coli. I. Adaptation and divergence during 2,000 generations. Am. Nat. 138, 1315-1341 (1991)

Supplementary Information is linked to the online version of the paper at www.nature.com/nature.

Acknowledgements We thank T. F. Cooper, M. R. Goddard and D. Refardt for discussion; M. Gray, A. Hurman and G. E. M. Lamers for technical assistance; M. Ackermann, P. M. Brakefield, T. Fukami, S. Rossell and B. J. Zwaan for comments on the manuscript; T. J. M. Van Dooren for statistical advice; F. Bertels for computational analysis of Solexa data; and E. Libby for theoretical insight. This work was supported by the Marsden Fund Council from government funding administered by the Royal Society of New Zealand. H.J.E.B. is supported by a Veni Fellowship from The Netherlands Organisation for Scientific Research (NWO). J.G. was supported by a Bright Futures Scholarship from the New Zealand Foundation for Research, Science and Technology. C.K. was supported by a Feodor Lynen Fellowship from the Alexander von Humboldt Foundation, Germany. G.C.F. is supported by a Postdoctoral Fellowship from the New Zealand Foundation for Research, Science and Technology.

Author Contributions H.J.E.B. and P.B.R. conceived the research and wrote the manuscript. H.J.E.B. conducted the main selection experiment, examined the relation between cell and colony morphology, investigated reversible switching, performed genome analysis and contributed to allelic replacements. J.G. determined $\mathrm{Cap}^{+}$cell proportions, performed transposon mutagenesis and integration-site identification, confirmed and ordered the mutations, performed allelic replacements, and contributed to fitness assays. C.K. and G.C.F. performed fitness assays. All authors commented on the manuscript

Author Information Reprints and permissions information is available at www.nature.com/reprints. Correspondence and requests for materials should be addressed to H.J.E.B. (h.j.e.beaumont@biology.leidenuniv.nl). 


\section{METHODS}

Strain and medium. P. fluorescens SBW25 (ref. 23) was grown at $28^{\circ} \mathrm{C}$ in $25-\mathrm{ml}$ glass microcosms containing $6 \mathrm{ml}$ King's Medium B with loose caps, and on King's Medium B-agar plates for $48 \mathrm{~h}$.

Selection regime. Populations were founded with approximately $10^{7}$ cells from $-80^{\circ} \mathrm{C}$ glycerol stocks prepared from cells derived from a single colony. For the first round, populations were grown in static microcosms and propagated by transfer of a mixed sample $(6 \mu \mathrm{l})$ to a fresh microcosm at 72 -h intervals of approximately ten generations. Parallel with each transfer, samples were spread onto plates to screen for colonies with a different morphology (approximately 1,000 screened, 500 minimum). Fuzzy spreader genotypes ${ }^{17}$ did not respond to selection (H.J.E.B., unpublished observations) and were omitted. Upon detection of new colony types, a single colony of the numerically dominant new type was streaked to single colonies on a control plate to confirm heritability of colony morphology, and to ensure a single-cell bottleneck. Emergence of a colony with a different morphology marked the end of a round. Cells of these colonies were taken from control plates, grown in a shaken microcosm $(16 \mathrm{~h})$ and stored at $-80^{\circ} \mathrm{C}$. Fifteen additional selection rounds (alternately shaken at 150 r.p.m. and static) were performed, each founded with the cells from the colony selected in the previous round. Control lines were continually selected in shaken microcosms (for the same number of rounds as the cognate selection lines) and maintained under stabilizing selection for the ancestral colony type by continual selection of a numerically dominant smooth ${ }^{17}$ colony.

Fitness assays. Cells taken from single colonies (grown from $-80{ }^{\circ} \mathrm{C}$ stocks) were pre-conditioned by cultivation in shaken microcosms for $24 \mathrm{~h}$. Competitions were inoculated with approximately $5 \times 10^{6}$ cells of each competitor, and incubated for $72 \mathrm{~h}$. Competitor frequencies were determined by plating at $0 \mathrm{~h}$ and $72 \mathrm{~h}$. Genotypes were distinguished by colony morphology or a neutral marker ${ }^{29}$. Relative fitness was expressed as the ratio of Malthusian parameters $^{30}$. 